\title{
An Ontology-Based Focused Crawler
}

\author{
Lefteris Kozanidis \\ Computer Engineering and Informatics Department, Patras University, 26500 Greece \\ kozanid@ceid.upatras.gr
}

\begin{abstract}
In this paper we present a novel approach for building a focused crawler. The goal of our crawler is to effectively identify web pages that relate to a set of predefined topics and download them regardless of their web topology or connectivity with other popular pages on the web. The main challenges that we address in our study concern the following. First we need to be able to effectively identify the pages' topical content before these are fully downloaded and processed. Thereafter, we need to obtain a well-balanced set of training examples that the crawler will regularly consult in its subsequent web visits.
\end{abstract}

\section{Introduction}

Web search engines are the entry point to the web for millions of people. In order for a search engine to be successful towards offering web users with the piece of information they are looking for, it is vital that the engine's index is as full and qualitative as possible. Considering the billions of pages that are available on the web and the evolution of the web population, it becomes evident that building a search engine to fit all purposes might be an extremely tedious task. To alleviate both search engine users and search engine developers from the burden of going over large volumes of data, researchers have proposed the implementation of vertical search engines; each containing specialized indices and thus serving particular user needs.

In this paper, we address the problem of building qualitative vertical search engines and we focus on the first step towards this pursuit, which concerns the implementation of a focused web crawler. The motivation of our study is to design a web crawler that will be able to focus its web visits on particular pages that are of interest to the vertical search engine users. The main problem that we have to overcome in the process of building a focused crawler lies on the effective identification of topic-relevant web pages and their exploitation in a way that will facilitate the crawler's decision making policy. More formally, consider a web crawler that navigates in the web space and looks for resources to download, simply by relying on their links. In the simplest case the crawler will download every resource it comes across regardless of the resource's content, quality of usefulness to the search engine users. In case though the crawler is intended to feed a vertical engine's index it must focus every web visit on the page's content so as to ensure that only qualitative and interesting pages will be downloaded. Based on the above, we realize that building a focused crawler is a complex yet vital task if we want to equip search engines with valuable indices that will be able to serve specific user requests and facilitate web users experience successful web searches. To accomplish the above 
goal we propose a novel approach for the design and implementation of a focused crawler that leverages a number of resources and tools from the NLP community in order to compile a rich knowledge base that the crawler will consult for making decisions about whether to download a given page, what is the download priority of every page it comes across and what should be the update policy that the crawler will need to adopt for ensuring that the topical focused index remains fresh. Before delving into the details of our method, let's first outline the current knowledge on the field and present the most widely known attempts of other researches who study the focused crawling problem. In Section 3, we present our proposed methodology, we describe the individual components of our focused crawling module and we outline our plans for future work.

\section{Related Work}

Researchers have studied the focused crawling problem in the past and proposed a number of algorithms. For instance in [5] the authors propose the Fish Search algorithm, which treats every URL as fish whose survivability depends on visited page relevance and server speed. In [7] the Shark-Search algorithm improves FishSearch as it uses vector space model in order to calculate the similarity between visited page and query. [3] suggested the exploitation of a document taxonomy for building classification models and proposed two different rules for link expansion, namely the Hard Focus and the Soft focus rules. In [4] two separate models are used to compute the page relevance and the URL visiting order, i.e. the online training by examples for the URL ranking and the source page features for deriving the pages' relevance. Later [6] introduced a new focused crawling approach based on a graphoriented knowledge representation and showed that their method outperforms typical breath-first keyword or taxonomic based approaches. [1] developed a rule-based crawler which uses simple rules derived from inter class linkage patterns to decide its next move. Moreover their rule-based crawler support tunnelling and try to learn which off topic pages can eventually lead to high quality on-topic pages. This approach improves the crawler in [2] in both in harvest rate and coverage.

\section{Our Proposed Focused Crawling Methodology}

To design a topical focused crawler, we rely on two distinct yet complementary programs: (i) a classifier that integrates a topical ontology in order to firstly detect the topical content of a web page and thereafter compute the relevance of the page to the ontology topic in which it has been assigned and (ii) a passage extraction algorithm that given a classified web page, it semantically process its content in order to extract the text nugget that is the most semantically close to the page's topical content. Based on both the topic relevance values and the topic similar extracts of a large number of web pages, we can built a knowledge base of training examples that the crawler will periodically consult in its web walkthroughs in order to judge whether a new (i.e. yet un-visited) page should be downloaded and if so in which priority order. Figure 1 
illustrates the general architecture of our focused crawler. As the figure illustrates the pre-requisites of our focused crawler are: a topical ontology, a classification module, a passage extraction algorithm and a generic crawling infrastructure. Let's not turn our attention to the description of how the above systems can be put together in order to turn a generic crawler into a topical focused one. Given a set of web pages, we preprocess them in order to derive a set of thematic keywords from their contents. For keywords extraction one could employ any of the well-known techniques in the literature. However, in our current study we experiment with the lexical chaining approach [2], which explores the WordNet lexical ontology [9] in order to generate sequences of semantically related term, the so-called thematic terms.

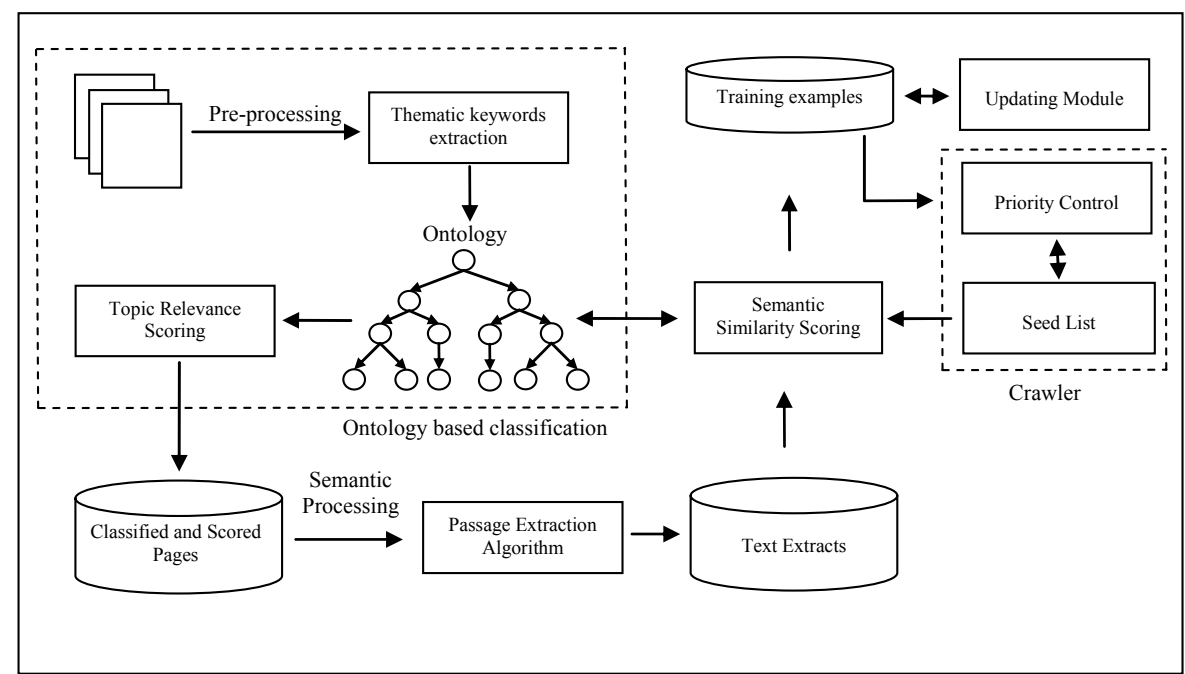

Fig. 1. Focused Crawler Architecture.

Having extracted a number of keywords from the page's contents, we employ a topical ontology and we map the pages' keywords to their corresponding ontology nodes in order to compute an appropriate ontology topic for representing the page's thematic content. Again the ontology that could be used for web pages' classification might be any ontology one would like to use. In our work, we are experimenting with the TODE topical ontology [8] that has proved to be richly encoded and useful for web pages' classification. Based on the ontology nodes that match the page's thematic keywords we can compute the degree to which every ontology topic is to likely represent the page's content as the fraction of the page's thematic terms that match an ontology topic $\mathrm{T}$, formally given by

$$
\operatorname{Topic} \text { Relevance }(p)=\frac{\mid \text { thematic keywords in p matching T } \mid}{\mid \text { thematic keywords in } p \mid}
$$

Based on the above formula we estimate the probability with which every page belongs to an ontology topic. Following web pages' classification to the ontology topics, we employ a passage extraction algorithm in order to extract from the pages 
contents the text nugget that is semantically closest to the identified topics. The passage extraction algorithm segments the pages' textual content into passages of 50 consecutive terms and relies on the semantic similarity values that the terms in every passage exhibit to the identified page's topic. In particular, to estimate the semantic similarity between a given ontology topic and the terms in the passage of a page that has been assigned to this topic we work as follows. We map all terms inside every page to the ontology and we apply the Wu and Palmer [10] similarity measure, which computes the degree to which passage terms semantically relate to the ontology concepts that represent topical categories. Having computed pared similarity values between passage terms and the ontology topics we take the average similarity values between a passage and a topic. Thereafter from all the page's passages we rely on the one that has the highest overall similarity to the most relevant page topic and we use it as a training example of the crawler's knowledge base. This way, we generate a number of example passages that semantically correlate to specific ontology topics. These examples are given as input to a core crawling module together with a seed list of URLs to be downloaded. Every time the crawler reaches to a page absent from the seed list, it downloads a $10 \%$ portion of its content in which it looks for patterns that match the crawler's training examples. If such patterns are found the page is downloaded. Depending on the semantic correlation that the detected patterns exhibit to the pre-defined topics the crawler's priority policy can be fine-tuned so as to ensure index freshness, quality and completeness.

Currently, we are experimenting with the crawler's effectiveness in performing complete topical crawls and we are testing its accuracy in building priority cues that are topic specific and resources affordable.

\section{References}

1. Altingovde I.S., Ulusoy O.: Exploiting interclass rules for focused crawling, In: Intelligent Systems, IEEE p66- 73 (Dec. 2004)

2. Barzilay R.: Lexical chains for text summarization. Master's Thesis, (1997)

3. Chakrabarti S., M. van den Berg, and B. Dom.: Focused Crawling: A new approach to Topic-Specific Web Resource Discovery. Computer Networks 31(11-16), (1999)

4. Chakrabarti S., Punera K., and Subramanyam M.: Accelerated focused crawling through online relevance feedback In WWW2002, Honolulu, Hawaii USA ACM, (May 7-11, 2002)

5. De Bra P., Houben G., Kornatzky Y., and Post R.: Information Retrieval in Distributed Hypertexts, In: Proceedings of the 4th RIAO Conference, 481 - 491, New York, (1994)

6. Ehrig M., Maedche A.: Ontology-focused Crawling of Web Documents. In the ACM Symposium on Applied computing. (2003)

7. Hersovici, M., Jacovi M., Maarek Y., Pelleg D., Shtalhaim M., and Ur S.: The shark-search algorithm - An application: Tailored Web site mapping. In Proc. 7th Intl. World-Wide Web Conference, Brisbane, Australia, (April 1998)

8. Stamou S., Krikos V., Kokosis P., Ntoulas A.: and Christodoulakis D. Web directory construction using lexical chains. In: the 10th NLDB Conference, 138-149, (2005)

9. WordNet. Available at http://www.cogsci.princeton.edu/ wn

10. $\mathrm{Wu}$ X. and Palmer M.: Web semantics and lexical selection. In the 32nd ACL Meeting, (1994) 